\title{
Research on Fault Recovery of Construction Engineering Distribution Network Based on Genetic Algorithm
}

\author{
Hongyi Guo \\ Southwest Jiaotong University Hope Collge, School of Civil Engineering, Urban-Rural Construction Depart \\ ment Sichuan Chengdu 610400
}

Keywords: Genetic algorithm; fault recovery; basic principle; objective function; chromosome coding

\begin{abstract}
In the process of building engineering distribution network fault recovery, the application of genetic algorithm can not only help the staff to get the best network structure reorganization strategy quickly, but also get many other relatively good solution strategies for the staff. Efficient recovery of distribution network operation provides a large amount of reference data. This paper expounds the principle and advantages of genetic algorithm, discusses the mathematical model of fault recovery in distribution network based on genetic algorithm and discusses the specific implementation of genetic algorithm for reference.
\end{abstract}

In recent years, various regions in China have been expanding the scale of the city, and various construction projects have been invested in the construction process. At the same time, the continuous improvement of power grid technology has made the construction engineering distribution network system more complicated, and the frequency of distribution network failures has also increased. The staff needs to start from the aspects of electrical relations and the topology of the distribution network, and effectively deal with various distribution network faults in the first time, thus ensuring the operation effect of the distribution network. Therefore, it is necessary to conduct an in-depth study on the failure recovery strategy of the construction project distribution network.

\section{Principles and advantages of genetic algorithms}

\subsection{The basic principles of genetic algorithms}

The so-called genetic algorithm is based on the theory of biological evolution, through the simulation of the reproductive process of the biological group - the survival of the fittest reproduction, to achieve an effective way to optimize the problem. Genetic algorithms have been widely used in engineering optimization, computer and economic related industries. It is similar to natural evolution to some extent and uses genes acting on chromosomes to seek excellent chromosomes to solve problems. The genetic algorithm only needs to evaluate each chromosome generated by the algorithm and at the same time, the chromosome selection is based on the fitness value so as to provide more breeding opportunities for more adaptive chromosomes ${ }^{[1]}$.

The basic principle of genetic algorithm is: firstly, a certain number of numerical codes, namely chromosomes, are generated in a random manner to serve as the initial population. Secondly, the fitness function is used to obtain the corresponding value to evaluate each individual, and the relatively low fitness is effectively eliminated. Thirdly, individuals with higher fitness were selected to participate in the genetic operation, and the set of individuals after the genetic operation formed a new generation of population. Finally, a new round of evolution is started on the basis of new species.

\subsection{Advantages of genetic algorithm}

Firstly, the operating object of the genetic algorithm is the coding of the variable, not the variable itself. Secondly, the traditional optimization algorithm is based on a single point to solve, often 
falling into the local solution, while the genetic algorithm is based on the combination of multiple points to solve the population exploration problem. Thirdly, the calculation of the adaptive value in the genetic algorithm depends on the objective function, and then the individual is evaluated with the value without any additional information or other derivation. Fourth, traditional optimization algorithm to search the solution by means of exhaustion or random, and genetic algorithm is carried out within the solution space has the high efficiency of heuristic search, although it is also a random search, but its essence is to improve the quality of the solution of the search space for the direction of the search method, the characteristic of directional search efficiency was improved greatly. Fifth, the traditional optimization algorithm blindly searches through trial or exhaustive search, while the genetic algorithm is a sufficient search in the solution space. It takes fitness as the basic basis for selection, so the search efficiency of the genetic algorithm is much higher than that of other optimization algorithms.

\section{Mathematical model of distribution network fault recovery based on genetic algorithm}

From the point of view of the open loop radiation characteristics of the construction project distribution network, the mathematical model of restoration and reconstruction can be defined according to the application situation and purpose, which is generally described as follows:

$$
\left\{\begin{array}{c}
\text { Obj min } \quad f \quad(S, X) \\
\text { s.t. } h \quad(S, X)=0 \\
g \quad(S, X) \leq 0 \\
\quad S \in T
\end{array}\right.
$$

In this equation, $f(S, X)$ represents the objective function of the mathematical model, s represents the control variable, $\mathrm{x}$ represents the state variable, $\mathrm{h}$ represents the equality constraint, g represents the inequality constraint and $\mathrm{T}$ represents the solution space of the problem sought.

\subsection{Objective function of mathematical model}

The research goal of this paper is to maximize the load in the recovery area after a single network failure occurs. The objective function is:

$$
\max F=\sum_{i=1}^{n} \lambda_{i} S_{i} l_{i}
$$

In the formula, $\mathrm{i}$ stands for the number of the $\mathrm{i}$-th branch, $\mathrm{n}$ stands for the total number of branches, $\lambda$ istands for the weight of the i-th branch, Si stands for the injection power of the i-th branch, and li stands for the $i$-th branch State, turn on li=1, otherwise li=0.

\subsection{The constraint conditions of mathematical model}

First, the power flow constraint needs to recalculate the power flow distribution after each change of the structure, and then push back to the generation method by layering.

Second, line capacity constraints:

$$
S_{i}<S \max
$$

In the equation, Si and Smax respectively represent the calculated value and the maximum allowable value of the power flowing through each line.

Third, the node voltage constraint:

$$
V_{i \min }<V_{i}<V_{i \max }
$$

In the equation, Vimin and Vimaxrespectively represent the lower limit and the upper limit of the node voltage. 
Fourth, the radial network constraint: after reconstruction, the radial structure of the distribution network must be maintained, and the ring network cannot appear.

In the failure recovery of construction engineering distribution network, the network structure should be inspected before each change of its structure. If there is an island or closed loop, it is an illegal structure and should be further processed until the radial structure is formed ${ }^{[2]}$. If the network structure meets the requirements, the power flow calculation is required before the inequality constraint test can be performed and the objective function calculation is performed. Therefore, the inequality constraint can be added to the objective function as a cross-border penalty function term to effectively simplify the processing flow.

\section{Implementation of genetic algorithm in fault recovery of distribution network in construction engineering}

\subsection{The main process of the genetic algorithm}

The fault recovery of the distribution network of the construction project is to isolate the faulty branch and find the combination of the switch states that meet the constraints and optimize the target within the new network structure. In order to satisfy the constraint conditions of radial network, the integrated loop of all switch state combinations should be opened. Therefore, the first task is to find all the loops in the isolated network structure, and then initialize the data with the same branching and node-related parameters to facilitate the optimization and reorganization of subsequent network structures.

\subsection{Chromosome coding}

Genetic algorithm (ga) usually performs a series of genetic operations to recombine the specific structural form of individuals in the population, continuously searching for the decoupling stock similarity between individuals, and then gradually searching for the best solution. Generally, due to the robustness of the genetic algorithm, the coding requirements are relatively loose, and the coding strategies or coding methods in the algorithm greatly affect the genetic operations or crossover operations. In most cases, the encoding form has a decisive effect on the crossover operation.

First, the existing chromosome code. In many literatures, chromosome coding usually refers to the switch state in the network as 0 and 1 , the first bit of chromosome represents each switch, 0 represents the condition of tripping, 1 represents the condition of closing, the situation of each branch is a chromosome, its length is the number of switches in the network. This kind of scheme is clear at a glance, but it cannot fully reflect the characteristics of the distribution network. In the process of running the genetic algorithm, there will be many infeasible solutions, which will have a great impact on its calculation efficiency.

Second, improved chromosome coding. The power point and $\mathrm{T}$ contact must be in the closed position, without paying attention to the situation of the terminal point. Therefore, in the process of encoding the chromosome, this part of the node can be effectively deleted. That is to say, the chromosome is composed of the t-junction and the node outside the power point, and there cannot be repeated switches in its switch set.

\subsection{Generation of initial population}

By randomly generating matrix of 44 columns and 10 rows, only elements of 0 and 1 exist in this matrix to represent different states of distribution network in various situations. Generation of such matrix makes understanding more convenient. In order to ensure the normal operation of the radiation network during supply, any two power sources cannot be connected. The method used here is as follows: first, all dual power supply that may be connected should be stored; second, all chromosomes in the initial population are reasonably judged. If the power supply requirements of the radiation network can’t be met, either operable switch in two power sources should be turned on. 


\subsection{The concrete operation of genetic algorithm}

The operation steps of genetic algorithm can be divided into four steps: selection, crossover, mutation and repair. In the process of selection operation, the best individual preservation method and fitness proportion selection method should be effectively combined. The first step is to arrange the order of the population calculated by the objective function from small to large. The first chromosome is the best choice and should be retained. Step 2: copy Chromosome 2,3,4 once, add them to 5,6,7, and eliminate the worst one. Step 3: put the original 5,6,7 into 8, 9 and 10. This method is very simple and easy to operate.

The selected individuals are intersected at two points, and the number of bodies to be crossed is calculated based on the crossing rate at first. The individuals are paired off in a random fashion, then the parts that need to be crossed are generated in a random manner and the corresponding positions of the matched individuals are exchanged.

The new population generated after crossing is randomly mutated, that is, to calculate the number of individuals who need variation according to the mutation rate. The individuals who need variation and their variation parts are obtained in a random way, and then the gene value of this part is changed. The crossover and mutation should be fully repaired to avoid the infeasible solutions

\section{Conclusion}

In short, genetic algorithm can carry out comprehensive parallel search, and at the same time, it can constantly adjust its search range with the goal of optimal solution in the search process, which is very suitable for searching optimal solution. Therefore, the staff must grasp the basic principle of genetic algorithm and hereby scientifically establish the mathematical model of fault recovery of construction project distribution network. Through the effective implementation of genetic algorithm, the operation of distribution network should be restored as soon as possible, so as to improve the efficiency and quality of construction project distribution network operation.

\section{References}

[1] Pengcheng Liu. Study on fault location and recovery reconstruction of intelligent distribution network[D]. North China Electric Power University(Beijing), 2016.

[2] Hongpeng Li. Study on fault recovery and reconstruction of active distribution network[D]. Shenyang Agricultural University, 2016. 